\title{
Assessment of Culprit Lesion of Optical Coherence Tomography in Patients With Acute Myocardial Infarction: a Case Report
}

\author{
Han-Joon Bae, MD \\ Division of Cardiology, Department of Internal Medicine, Daegu Catholic University Medical Center, Daegu, Republic of Korea
}

\section{ABSTRACT}

In general, patients with acute myocardial infarction (AMI) have severe stenosis secondary to a superimposed thrombus at the event. Optical coherence tomography (OCT) is a useful imaging tool for patients with AMI. This allows us to identify the site of ruptured plaque, erosion of fibrous cap, and characteristics of stenotic lesion. In this case, we present the difference of the ruptured cavity and obstructed lesion.

\section{INTRODUCTION}

Rupture of vulnerable plaque with thrombi is a major cause of acute coronary syndrome (ACS) [Ino 2011]. The pathological features of vulnerable plaques include a thin fibrous cap with macrophage infiltration and a large lipid core [Virmani 2000; Falk 1995]. Optical coherence tomography (OCT) is a high-resolution imaging modality, that is about 10 times higher than intravascular ultrasound. OCT shows excellent imaging to find plaque rupture, thin-cap fibroatheromas (TCFAs), and intracoronary thrombus [Kubo 2007; Kubo 2010]. We used OCT to find the culprit lesion.

\section{CASE REPORT}

A 65-year-old female patient came in, complaining about severe squeezing chest pain. She had a medical history that showed partial gastrostomy due to gastric cancer 12 years ago and total thyroidectomy due to thyroid cancer 5 years ago. She had no cardiovascular risk factors. An electrocardiogram revealed elevation of the ST-segment elevation in II III aVF.

Coronary angiography showed significant stenotic lesion of the distal right coronary artery (RCA) (Figure 1A). After $2.0 \mathrm{~mm}$ ballooning, we proceeded to 6 French catheter thrombosuction, there was a huge red thrombus (Figure 1B). We continued optical coherence tomography (OCT, Lightlab Imaging/St. Jude Medical, Westford, MA), because

Received fuly 29, 2019; accepted October 15, 2019.

Correspondence: Han-Foon Bae, Division of Cardiology, Department of Internal Medicine, Daegu Catholic University Medical Center, Daegu, Republic of Korea 33, Duryugongwon-ro 17-gil, Nam-gu, Daegu (42472), Republic of Korea; 82-53-650-3611; fax: 82-53-623-7507 (e-mail: guanwoo@gmail.com). of the evaluation. But OCT finding of the distal RCA lesion revealed an intact cap lesion, and there was no definite plaque rupture or erosion (Figure $2 \mathrm{C}, \mathrm{D})$. On the other side of the proximal RCA, where was plaque disruption (Figure 2B, E, F, G).
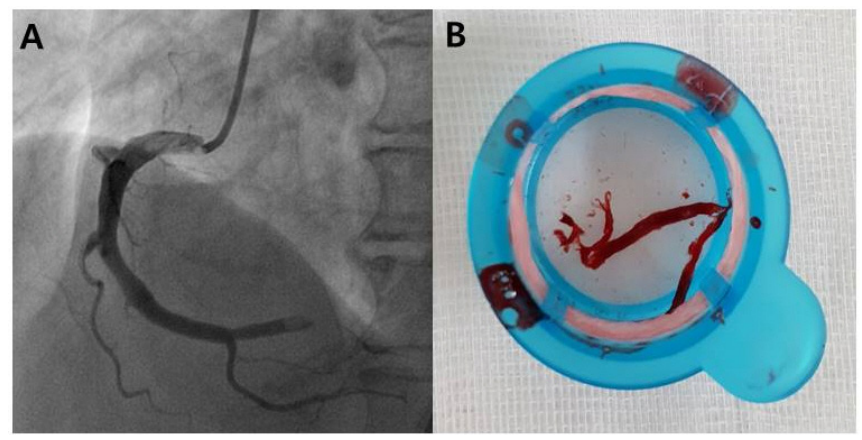

Figure 1. (A) The initial angiography finding of the RCA; (B) Thrombus.

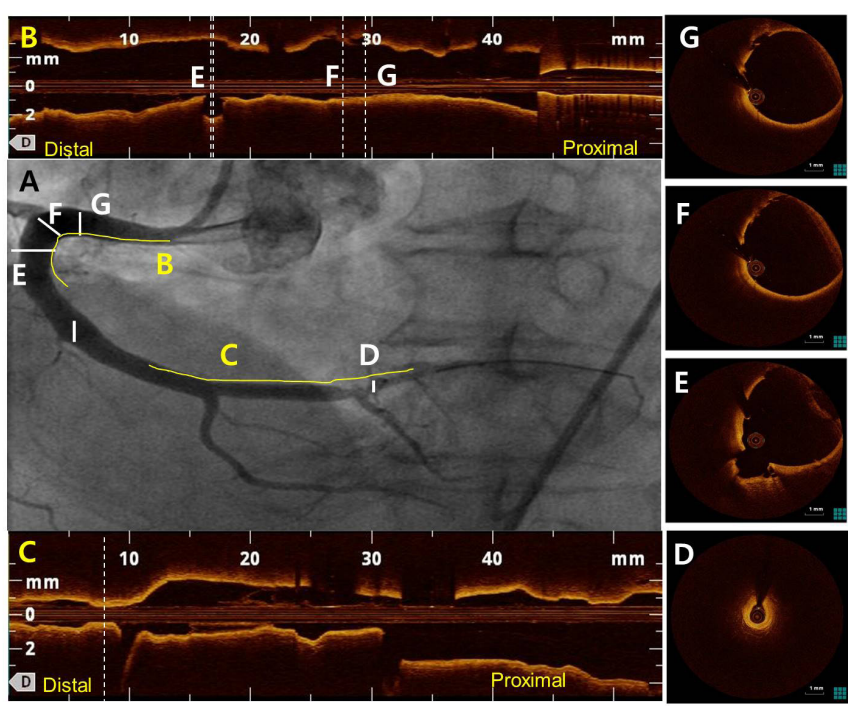

Figure 2. Coronary angiography and optical coherence tomography (OCT) of the right coronary artery (RCA); (A) Angiography image of the post ballooning and post thrombosuction; (B) OCT image of proximal RCA; (C) OCT image of distal RCA to postero-lateral (PL) branch; (D) OCT image of the tight stenotic lesion at PL branch; (E) OCT image of ruptured plaque; (F) OCT image of plaque erosion; (G) OCT image of ruptured plaque. 


\section{DISCUSSION}

The ACS is a rupture of an atherosclerotic lesion that contains a large necrotic core, and a thin fibrous cap causes acute luminal thrombosis and sometimes acute luminal obstruction by platelet aggregation [Choi 2010]. The high resolution of OCT is useful for the evaluation of vulnerable coronary plaques in patients with AMI. The fibrous cap disruption, fibrous cap erosion, intracoronary thrombus, and thin-cap fibroatheroma (TCFA) could be identified more frequently by OCT in comparison with IVUS or CAS [Kubo 2007]. Patients with ACS caused by ruptured plaques had increased pan-coronary vulnerability and were more likely to have a greater lipid arc, higher incidence of thrombus, and smaller minimum lumen area of the culprit lesion, suggesting that the morphology of the plaque rupture may influence whether it heals asymptomatically or causes an adverse cardiovascular event [Kubo 2010; Shimamura 2014].

In our case, angiographical obstruction of distal RCA was observed. However, OCT did not show the plaque rupture at distal RCA. We found the ruptured plaque at proximal RCA.

\section{REFERENCES}

Choi SY, Mintz GS. 2010. What Have We Learned About Plaque
Rupture in Acute Coronary Syndromes? Curr Cardiol Rep 12:338-43.

Falk E, Shah PK, Fuster V. 1995. Coronary plaque disruption. Circulatio 92:657-71.

Ino Y, Kubo T, Tanaka A, et al. 2011. Difference of culprit lesion morphologies between ST-segment elevation myocardial infarction and nonST-segment elevation acute coronary syndrome: an optical coherence tomography study. JACC Cardiovasc Interv 4:76-82.

Kubo T, Akasaka T. 2010. Optical coherence tomography imaging: current status and future perspectives: Current and future developments in OCT. Cardiovasc Interv Ther 25:2-10.

Kubo T, Imanishi T, Kashiwagi M, et al. 2010. Multiple coronary lesion instability in patients with acute myocardial infarction as determined by optical coherence tomography. Am J Cardiol 105:318-22.

Kubo T, Imanishi T, Takarada S, et al. 2007. Assessment of culprit lesion morphology in acute myocardial infarction: ability of optical coherence tomography compared with intravascular ultrasound and coronary angioscopy. J Am Coll Cardiol 50:933-9.

Shimamura K, Ino Y, Kubo T, et al. 2014. Difference of ruptured plaque morphology between asymptomatic coronary artery disease and non-ST elevation acute coronary syndrome patients: an optical coherence tomography study. Atherosclerosis 235:532-7.

Virmani R, Kolodgie FD, Burke AP, et al. 2000. Lessons from sudden coronary death: a comprehensive morphological classification scheme for atherosclerotic lesions. Arterioscler Thromb Vasc Biol 20:1262-75. 